\title{
Effects of dipole position, orientation and noise on the accuracy of EEG source localization Kevin Whittingstall*1, Gerhard Stroink ${ }^{1}$, Larry Gates ${ }^{2}$, JF Connolly ${ }^{3}$ and Allen Finley 4
}

\author{
Address: ${ }^{1}$ Department of Physics, Dalhousie University, Halifax NS, Canada, ${ }^{2}$ Department of Radiation Oncology, QEII Health Centre, Halifax, \\ Canada, ${ }^{3}$ Department of Psychology, Dalhousie University, Canada and ${ }^{4}$ Department of Anaesthesia, Dalhousie University, Halifax, Canada \\ Email: Kevin Whittingstall* - kwhittin@dal.ca; Gerhard Stroink - stroink@is.dal.ca; Larry Gates - lgates@is.dal.ca; \\ JF Connolly - connol@is.dal.ca; Allen Finley - allen.finley@dal.ca \\ * Corresponding author
}

Published: 6 June 2003

BioMedical Engineering OnLine 2003, 2:14
Received: 16 January 2003

Accepted: 6 June 2003

This article is available from: http://www.biomedical-engineering-online.com/content/2/1/14

(C) 2003 Whittingstall et al; licensee BioMed Central Ltd. This is an Open Access article: verbatim copying and redistribution of this article are permitted in all media for any purpose, provided this notice is preserved along with the article's original URL.

\begin{abstract}
Background: The electroencephalogram (EEG) reflects the electrical activity in the brain on the surface of scalp. A major challenge in this field is the localization of sources in the brain responsible for eliciting the EEG signal measured at the scalp. In order to estimate the location of these sources, one must correctly model the sources, i.e., dipoles, as well as the volume conductor in which the resulting currents flow. In this study, we investigate the effects of dipole depth and orientation on source localization with varying sets of simulated random noise in 4 realistic head models.

Methods: Dipole simulations were performed using realistic head models and using the boundary element method (BEM). In all, 92 dipole locations placed in temporal and parietal regions of the head with varying depth and orientation were investigated along with 6 different levels of simulated random noise. Localization errors due to dipole depth, orientation and noise were investigated.

Results: The results indicate that there are no significant differences in localization error due tangential and radial dipoles. With high levels of simulated Gaussian noise, localization errors are depth-dependant. For low levels of added noise, errors are similar for both deep and superficial sources.

Conclusion: It was found that if the signal-to-noise ratio is above a certain threshold, localization errors in realistic head models are, on average the same for deep and superficial sources. As the noise increases, localization errors increase, particularly for deep sources.
\end{abstract}

\section{Background}

The localization of electrical activity on the basis of EEG recordings has found several applications in neuroscience. The location of this activity can be estimated from the calculation of inverse solutions in which the location, amplitude and orientation of a source are adjusted to obtain a best fit between the measured EEG's and the calculated potentials produced by the source. This calculation of the magnetic fields and electric potentials at the scalp due to a source in a head model is called the forward problem. As source model, the equivalent current dipole has been widely used as a source representing focal neural activity $[1,2]$. Current dipoles do, however, not only impress a current at the source location. They also cause currents to flow in the surrounding tissue, or, so-called volume currents. These currents are influenced by the shape and 
conductivity of the different tissues. Clearly, the accuracy with which sources can be localized will be affected by a number of factors including source-modelling errors, head modelling errors and noise (biological (background EEG, blinking, etc.) and electrical).

The concentric multi-sphere model is commonly used for modelling the head, although, its simplistic geometry can result in localization errors. Alternative numerical forward solution approaches account for the individual shape of the head layers. One of these approaches is the boundary element method (BEM), where the head model consists of realistically shaped closed layers with different conductivity values. Although this method still has some severe limitations, such as homogeneity and isotropy of the tissues involved, it enables us to replace the spherical model of the head with a more realistically shaped model for EEG source localization.

Several authors have explored the effects of the depth of a dipolar source on localization accuracy using a spherical head model as volume conductor [3-6]. Using an experimentally constructed realistically shaped head model for inverse calculations, Menninghaus et al. [7] considered both radial and tangential dipoles placed between 10 and $30 \mathrm{~mm}$ below the brain surface. They found localization errors in the order of 2-4 mm. However, these sources represent activity only near the surface of the brain, which does not represent deep electrical sources, such as those in clinical epilepsy studies $[8,9]$. Moreover, the effects of noise were not investigated. Yvert et al. [10] demonstrated the importance of dipole depth using a realistic head model with 32 electrodes for forward and inverse calculations, although the effects of noise were not investigated. In [11], the effects of simulated noise on dipole localization using realistic models were investigated. Here, localization errors were averaged over all dipole positions. It is left to be seen how noise affects dipoles of different depths and regions in realistically shaped head models.

The direction in which the source is orientated (i.e., tangential or radial to the cortical surface) may also play a role in how accurately it can be reconstructed. Previous theoretical studies have used spherical heads to investigate localization errors resulting from radial and tangential orientated dipoles $[7,12,13]$. Menninghaus et al. [7] reported higher localization errors for radial sources than tangential ones in a phantom study. However, the sources were limited to $30 \mathrm{~mm}$ below the cortical surface and only placed in the temporal region.

In this report, localization errors resulting from dipoles of different orientation and depth are investigated in simulated noisy environments using the same realistic head model for forward and inverse computations.
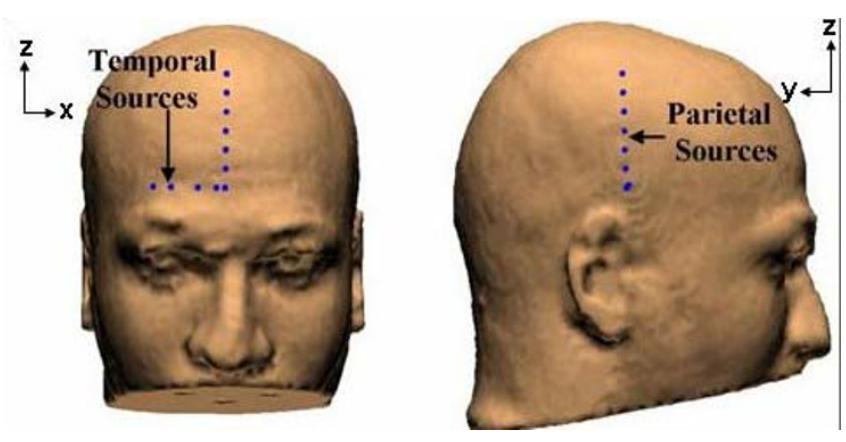

\section{Figure I}

Location of the parietal and temporal dipoles in a realistic head model. Only 5 of the 23 temporal and 7 of the 23 parietal dipole locations are shown. Each location is used to simulate the potentials of a tangential and radial dipole.

\section{Methods}

Twenty-three tangential and radial dipoles were evenly spaced along the $\mathrm{x}$ and $\mathrm{z}$ axis in the parietal and temporal regions of the brain. Overall, 92 source locations with varying depths and orientations were used for forward computations in four realistic head models (Figure 1). The coordinate system is defined via the preauricular points and the nasion of the subject. The positive $\mathrm{x}$-axis goes through the left preauricular point (PAL), the negative $y$ axis goes through the nasion and the positive $\mathrm{z}$-axis runs perpendicular to the intersection of the $\mathrm{x}$ and $\mathrm{y}$-axis, pointing from toe to head [14].

Distribution of the parietal and temporal dipoles was based on the distance between the origin and the intersection of the $\mathrm{z}$-axis and $\mathrm{x}$-axis with the brain surface, respectively. The dipole depth relates to the distance between the dipole location and where the positive $\mathrm{z}$-axis intercepts the brain surface for parietal sources, and negative $\mathrm{x}$ axis for temporal sources.

Four realistic head models were generated from four individual MRI scans using the boundary element method (BEM) as described in [15]. Each realistic model consisted of approximately 2000 triangles per compartment with a triangle edge length of 12,10 and $8 \mathrm{~mm}$ for the scalp, skull and brain regions, respectively. The data of each MRI scan consisted of 160 transverse slices, each containing $256^{*} 256$ voxels of $1 \mathrm{~mm}$ edge length. Time-varying signals via forward computations for the electric potentials were generated at 50 electrode sites on the scalp using the constant element approach of the BEM. All potentials were re-referenced to the average potential over all 50 electrodes. The 10-20 System was used for electrode placement. Each realistic head model contains a scalp, skull 


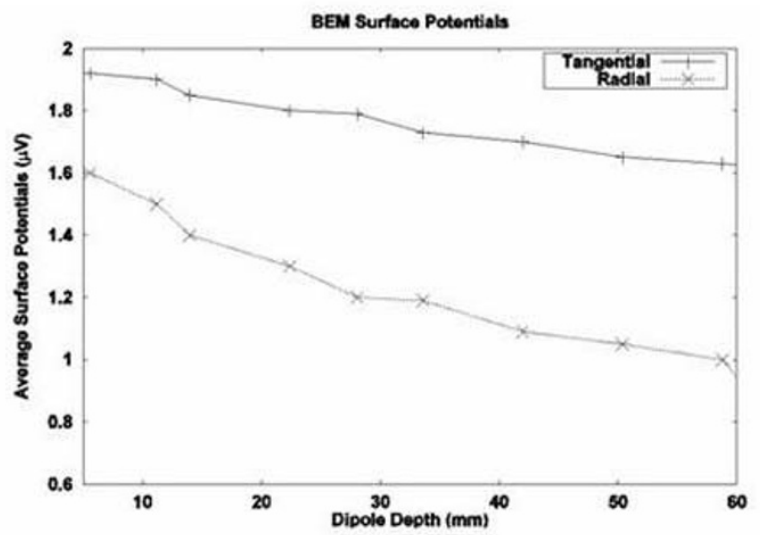

Figure 2

Simulated potentials due to tangential and radial dipoles at different depths. Potential values shown here are averaged over all 50 electrodes in one of the four realistically shaped head models. The potentials in the other head models show similar trends.

and brain compartment with conductivities set to 0.33 , 0.0042 , and $0.33 \mathrm{~S} / \mathrm{m}$, respectively, based on earlier literature [16-19]. Figure 2 shows the average grand field power of the simulated potentials over all 50 electrodes for one head model using radial and tangential dipole sources at different depths.

For each forward computation, random, zero-mean Gaussian noise with standard deviations of $0.04,0.06,0.1,0.2$, 0.5 , and $1 \mu \mathrm{V}$ were added to mimic normal, averaged EEG recordings and to observe its effect on dipole localization. The impact of noise on the simulated potentials can be expressed as the signal-to-noise ratio (SNR). SNR is defined as the ratio between the average root-mean squared (RMS) potential value over all electrodes and the RMS noise value used in this study, as shown in Table $1[18,19]$.

Dipole localization was carried out using the MUSIC algorithm $[20,21]$ on the same realistic head model from which the forward computations were made. For each noise level in Table 1, we used 20 sets of Gaussian noise distributions in order to obtain statistically meaningful results. With these noise sets, the localization procedure was repeated 20 times for each dipole location in each of the 6 noise levels. In all, 44,160 inverse solutions were performed. The resultant dipole locations were compared with the original setting of the test dipole locations to estimate the errors of source localization caused by deep and superficial sources at different noise levels. Errors are
Table I: Average SNR values of potentials resulting from all tangential dipoles. The SNR is defined as the root mean square (RMS) value of the simulated signal across all 50 electrode sites by the RMS simulated noise level. SNR values resulting from radial dipoles are slightly ( $5 \%)$ lower (not shown here).

\begin{tabular}{cc}
\hline RMS Noise $(\mu \mathbf{V})$ & Average SNR \\
\hline 0.035 & 40 \\
0.052 & 25 \\
0.087 & 18 \\
0.180 & 8 \\
0.430 & 4 \\
0.870 & 2 \\
\hline
\end{tabular}

defined as the square root of the sum of squares of the errors in the 3 axes between the position of the original dipole and calculated dipole. The results were then averaged for each dipole location, orientation and noise level. In our discussion, localization errors will be summarized as the average error of deep (35-65 mm) and superficial $(5-35 \mathrm{~mm})$ sources.

\section{Results}

The accuracy in terms of dipole localization error is depicted in Figure 3 for tangential and radial dipoles in the parietal and temporal regions. Each inverse calculation was carried out using the same volume conductor from which the simulated potentials originated; thus, the only modelling parameter affecting localization results was the addition of simulated random noise. Results with no added noise yielded no localization errors.

Overall, localization errors increased with dipole depth and level of simulated noise (Figure 3). This trend is similar for sources in both parietal and temporal regions. For RMS noise values below $0.180 \mu \mathrm{V}$ (8-40 SNR range), localization errors are only moderately influenced by the level of noise, regardless of source depth and orientation. However, as the noise level increases, differences in accuracy between superficial and deep sources begin to emerge. For noise levels above $0.43 \mu \mathrm{V}$ (2-4 SNR range), average localization errors were 2 and $4 \mathrm{~mm}$ for superficial and deep sources respectively. No significant differences in accuracy were found for sources in temporal and parietal regions. However, a slight difference in accuracy between tangential and radial sources (Figure 3 ) was seen. This difference is largest for deep sources.

\section{Discussion}

Figure 2 shows the mean RMS potential value at 50 electrode sites due to radial and tangential dipoles as a function of dipole depth. The difference in averaged potentials between radial and tangential sources is approximately 

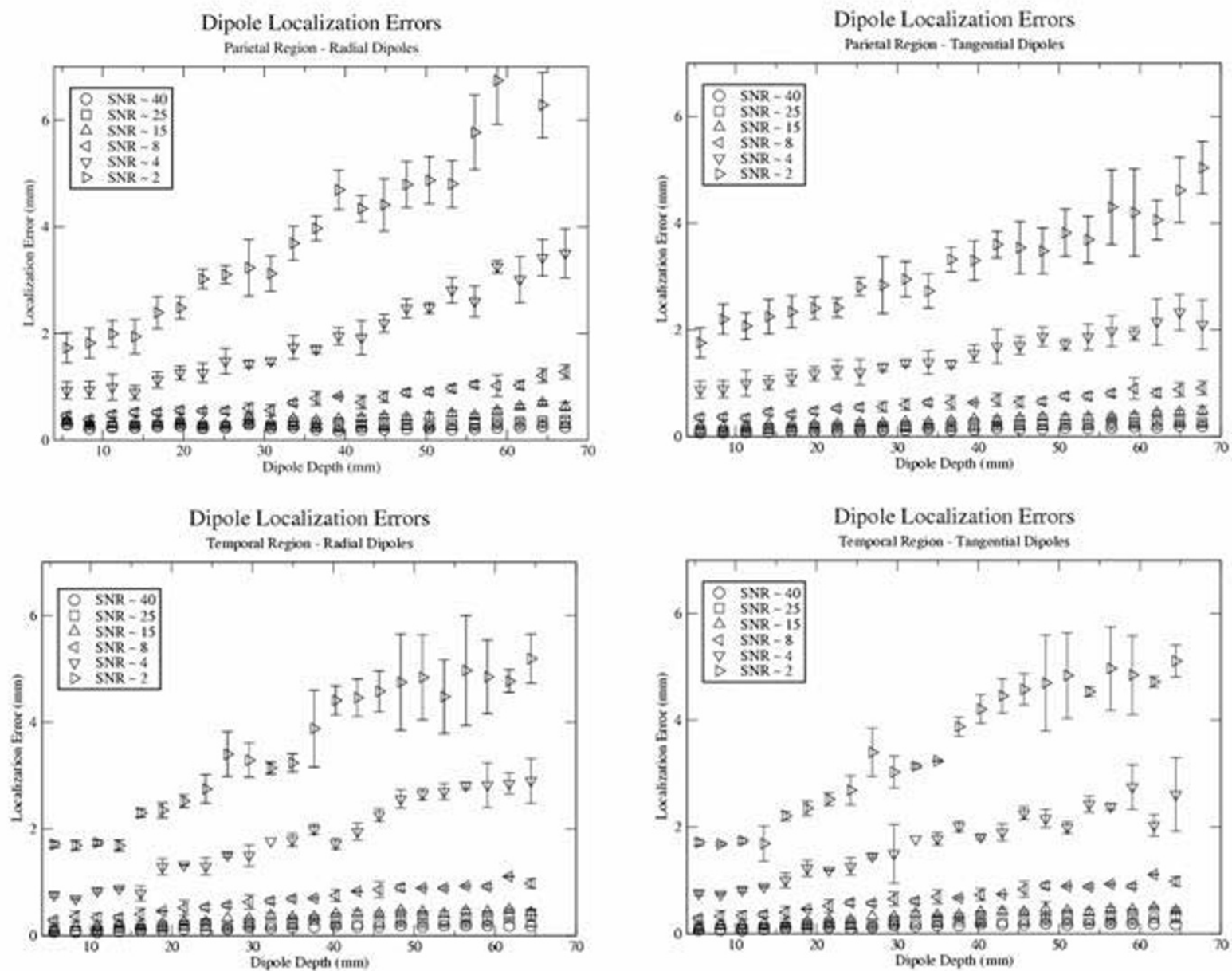

\section{Figure 3}

Average localization errors for simulated tangential and radial dipoles placed in the parietal region (top) and temporal region (bottom) for different depths and signal to noise ratios (SNR).

$0.4 \mu \mathrm{V}$ in shallow regions, and $0.6 \mu \mathrm{V}$ in deeper regions. Since the average of potentials arising from deep radial sources is smaller in magnitude than that of deep tangential sources, the effects of noise on these smaller potentials may make it, on average, more difficult for the inverse algorithm to deal with. This suggests that the slight decrease in localization accuracy for deep radial sources is not directly due to its orientation, but to its susceptibility to noise. However, since this difference in accuracy only appears at very low SNR values, these small differences are of little clinical value.

\section{Conclusions}

Given a set of measured scalp potentials, estimates of the location of neural activity are possible if a source and head model is specified. Sources can be located anywhere from near the surface of the cortex to deep within the brain stem. If the current dipole is used to model neural activity, its orientation can range from being parallel to perpendicular to the measuring surface. In this study, we examined the influences of dipole depth and orientation using an individual realistic head model for forward and inverse calculations. The findings are briefly summarized here:

- With the addition of simulated Gaussian noise $($ SNR $<4)$, average localization errors are depth dependent, i.e., superficial sources are more accurately estimated than sources deeper within the brain volume. For low values of added noise (SNR $>8$ ), localization errors are fairly constant for both deep and superficial sources. No significant 
difference in accuracy for radial or tangential sources is to be expected.

- In very noisy data (SNR $<4)$, a slight decrease in accuracy for radial sources can be expected, but not as much for tangential sources. However, EEG data of this quality is rarely used for source analysis.

It can therefore be concluded that, on average, noise plays a role in how accurately sources can be localized when using a realistic head model for forward and inverse calculations (for low levels of noise, the effects of dipole depth, and orientation are negligible). As the noise increases, localization accuracy begins to decrease, particularly for deep sources of radial orientation.

\section{Authors' contributions}

Author 1 (KW) constructed all realistic head models and carried out the entire simulation outlined in this manuscript. Author 2 (GS) assisted in the development of the simulation. Author 3 (LG) collected image data necessary for realistic head model construction. Author 4 (JFC) and Author 5 (AF) participated in the coordination of the study.

All authors have read and approved the final manuscript.

\section{Acknowledgements}

We would like to thank Dr. P. McGrath for his encouragement, as well as the anonymous reviewers for their helpful comments.

\section{References}

I. De Munck JC: Mathematical Dipoles are Adequate to Describe Realistic Generators of Human Brain Activity IEEE Trans Biomed Eng 1988, 35(II):960-6.

2. Schimpf PH: Dipole Models for the EEG and MEG IEEE Trans Biomed Eng 2002, 49(5):409-18.

3. Zanow and Frank : Realistically shaped models of the head and their application to EEG and MEG, Ph. D. Thesis University of Twente, The Netherlands 1997.

4. Thevenet $M$, Bertrand $O$, Perrin F, Dumont $T$ and Pernier J: The finite element method for a realistic head model of electrical brain activities: preliminary results Clin Phys Physiol Meas 199I, I 2(Suppl A):89-94.

5. Mosher JC, Spencer ME, Leahy RM and Lewis S: Error bounds for MEG and EEG source localization Electroencephalogr Clin Neurophysiol 1993, 86:303-21.

6. Roth : How well does a three-sphere model predict positions of dipoles in a realistically shaped head? Electroenceph Clin Neurophysiol 1993, 87: 175-184.

7. Menninghaus E, Lutkenhoner B and Gonzalez SL: Localization of a dipolar source in a skull phantom: realistic versus spherical model IEEE Trans Biomed Eng 1994, 4 I ( I 0):986-9.

8. Krings T, Chiappa KH, Cuffin BN, Cochius Jl, Connolly S and Cosgrove GR: Accuracy of EEG dipole source localization using implanted sources in the human brain Clin Neurophysiol 1999 , I I0(I):106-14.

9. Yoshinaga H: Benefit of Simultaneous Recording of EEG and MEG in Dipole localization Epilepsia 2002, 43(8):924-928.

10. Yvert B, Bertrand O, Echallier JF and Pernier J: Improved dipole localization using local mesh refinement of realistic head geometries: an EEG simulation study Electroencephalogr Clin Neurophysiol 1996, 99(I):79-89.
II. Vanrumste B: Comparison of performance of spherical and realistic head models in dipole localization from noisy EEG Med Eng \& Phys 2002, 24:403-18.

12. Ferguson AS and Stroink G: Factors affecting the accuracy of the boundary element method in the forward problem - I: Calculating surface potentials IEEE Trans Biomed Eng 1997, 44(I I): I I39-55.

13. Murro AM, Smith JR, King DW and Park YD: Precision of dipole localization in a spherical volume conductor: a comparison of referential EEG, magnetoencephalography and scalp current density methods Brain Topogr 1995, 8(2): 1 19-25.

14. Fuchs M, Kastner J, Wagner M, Hawes S and Ebersole JS: A standardized boundary element method volume conductor model Clinical Neurophysiology 2002, I I 3(I):702-7I 2.

15. Fuchs M, Drenckhahn R, Wischmann HA and Wagner M: An improved boundary element method for realistic volumeconductor modeling IEEE Trans Biomed Eng 1998, 45(8):980-97.

16. Homma S, Musha T, Nakajima Y, Okamoto Y, Blom S, Flink R and Hagbarth KE: Conductivity ratios of the scalp-skull-brain head model in estimating equivalent dipole sources in human brain Neurosci Res 1995, 22(I):5I-5.

17. Laarne PH, Tenhunen-Eskelinen ML, Hyttinen JK and Eskola $\mathrm{HJ}$ : Effect of EEG electrode density on dipole localization accuracy using two realistically shaped skull resistivity models Brain Topography 2000, I 2(4):249-54.

18. Wang $Y$ and Gotman J: The influence of electrode location errors on EEG dipole source localization with a realistic head model Clinical Neurophysiology 200 I, I I 2(9): 1777-80.

19. Cuffin BN: A method for localizing EEG sources in realistic head models IEEE Trans Biomed Eng 1995, 42(I):68-7I.

20. Mosher JC, Baillet S and Leahy RM: Multiple EEG source localization and imaging using multiple signal classification approaches Clinical Neurophysiology 1999, 16(3):225-38.

21. Van der Meij W, Huiskamp GJ, Wieneke GH, van Huffelen AC and van Nieuwenhuizen $O$ : The existence of two sources in rolandic epilepsy: confirmation with high resolution EEG. MEG and fMRI Brain Topography 200 I, I3(4):275-82.
Publish with BioMed Central and every scientist can read your work free of charge

"BioMed Central will be the most significant development for disseminating the results of biomedical research in our lifetime."

Sir Paul Nurse, Cancer Research UK

Your research papers will be:

- available free of charge to the entire biomedical community

- peer reviewed and published immediately upon acceptance

- cited in PubMed and archived on PubMed Central

- yours - you keep the copyright
BioMedcentral 